\title{
Fermi level alignment in molecular nanojunctions and its relation to charge transfer
}

\author{
Stadler, Robert; Jacobsen, Karsten Wedel
}

Published in:

Physical Review B Condensed Matter

Link to article, DOI:

10.1103/PhysRevB.74.161405

Publication date:

2006

Document Version

Publisher's PDF, also known as Version of record

Link back to DTU Orbit

Citation (APA):

Stadler, R., \& Jacobsen, K. W. (2006). Fermi level alignment in molecular nanojunctions and its relation to charge transfer. Physical Review B Condensed Matter, 74(16), 164105.

https://doi.org/10.1103/PhysRevB.74.161405

\section{General rights}

Copyright and moral rights for the publications made accessible in the public portal are retained by the authors and/or other copyright owners and it is a condition of accessing publications that users recognise and abide by the legal requirements associated with these rights.

- Users may download and print one copy of any publication from the public portal for the purpose of private study or research.

- You may not further distribute the material or use it for any profit-making activity or commercial gain

- You may freely distribute the URL identifying the publication in the public portal

If you believe that this document breaches copyright please contact us providing details, and we will remove access to the work immediately and investigate your claim. 


\title{
Fermi level alignment in molecular nanojunctions and its relation to charge transfer
}

\author{
R. Stadler and K. W. Jacobsen \\ Center for Atomic-Scale Materials Physics, Department of Physics, NanoDTU, Technical University of Denmark, \\ DK-2800 Kongens Lyngby, Denmark
}

(Received 28 July 2006; revised manuscript received 7 September 2006; published 20 October 2006)

\begin{abstract}
The alignment of the Fermi level of a metal electrode within the gap of the highest occupied molecular orbital (HOMO) and the lowest unoccupied molecular orbital (LUMO) of a molecule is a key quantity in molecular electronics, which can vary the electron transparency of a single-molecule junction by orders of magnitude. We present a quantitative analysis of the relation between this level alignment (which can be estimated from charging free molecules) and charge transfer for bipyridine and biphenyl dithiolate (BPDT) molecules attached to gold leads based on density functional theory calculations. For bipyridine the charge distribution is defined by a balance between electrostatic repulsion effects and the filling of the LUMO, where the molecule loses electrons to the leads. BPDT, on the other hand, gains electrons. As a direct consequence the Fermi level of the metal is found at the energetically higher end of the gap in the transmission function for bipyridine and at its lower end for BPDT.
\end{abstract}

DOI: 10.1103/PhysRevB.74.161405

PACS number(s): 73.63.Rt, 73.20.Hb, 73.40.Gk

Interest in electron transport in nanoscale contacts has recently intensified, because of (i) the advent of the technologically motivated field of molecular electronics, ${ }^{1-4}$ (ii) recent progress in experimental techniques for manipulating and contacting individual molecules, ${ }^{5-8}$ and (iii) the availability of first-principles methods to describe the electrical properties of single-molecule junctions. ${ }^{9-13}$ These latter methods are usually based on density functional theory (DFT) in combination with a nonequilibrium Green's function formalism. ${ }^{14}$

It has become clear through many studies ${ }^{15}$ that the conductances of molecular junctions can be entirely controlled by the positions of individual molecular levels. For example, in the case of a molecular contact consisting of a bipyridine molecule attached to $\mathrm{Au}$ leads it has been demonstrated ${ }^{16,17}$ that the transmission depends crucially on the position of the lowest unoccupied molecular orbital (LUMO) and that this position may vary significantly with, for example, the surface structure of the leads. In order to describe the transport in molecular junctions it thus becomes a key issue to determine factors controlling the lineup of molecular levels relative to the Fermi level of the metal. In the following we study two different molecular junctions which turn out to behave quite differently with respect to level alignment.

Figure 1 compares bipyridine and biphenyl dithiolate (BPDT) attached to gold electrodes with the same surface structure and the same bonding configuration (top panels). In both cases no molecular levels can be found close to the Fermi energy if the vacuum potentials from separate calculations for the molecules and the metal surface are set equal (middle lower panel). However, when the molecules are coupled to the electrodes, there are peaks in the transmission functions (left and right lower panels), where $E_{F}$ is crossed by their tails at different sides of a gap for the two different molecules. The exact position of these peaks determines the conductance which is defined by the value of the transmission function at $E_{F}$ and can vary between $0.03 G_{0}$ and $0.44 G_{0}$ for BPDT depending on the bonding configuration. What we shall show in the following is that the corresponding level shifts can be directly determined from an appropriately de- fined charge transfer between the metal surface and the molecule. The downshift of the bipyridine LUMO is, for example, associated with an electronic charge transfer from the molecule to the surface. This might at first seem counterintuitive since the downshift of the LUMO apparently leads to a slight occupation of this state (see Fig. 1), but still the net electron transfer is away from the molecule due to a combination of Pauli repulsion and screening effects as we shall demonstrate. For benzene dithiolate (BDT) and BPDT the alignment of molecular levels coupled to a $\mathrm{Au}$ (111) surface has previously been suggested to be linked to charge being transferred from the surfaces to the molecules. ${ }^{18}$

All electronic structure calculations in this study are performed using a plane wave implementation ${ }^{19}$ of DFT with an energy cutoff of $340 \mathrm{eV}$, where we used ultrasoft pseudopotentials, ${ }^{20}$ and a Perdew-Wang 1991 (PW91) parametrization for the exchange and correlation functional. ${ }^{21}$ The transmission functions of the molecular junctions in Fig. 1 were calculated using a general nonequilibrium Green's function formalism for phase-coherent electron transport, ${ }^{22}$ where both the Green's function of the scattering region and the self-energies describing the coupling to the semi-infinite electrodes were evaluated in terms of a basis consisting of maximally localized Wannier functions. ${ }^{23}$ In our calculations the supercells for the scattering region are defined by $3 \times 3$ atoms in the surface plane and contain three to four surface layers on each side of the molecule. We used a $4 \times 4$ grid for the $\mathbf{k}$-point integration in order to obtain well-converged results for the conductance. ${ }^{24}$

We investigated the variation of the energetic position of the lowest-lying molecular orbital (denoted MO1 in the following text) with respect to the metal's Fermi level, in dependence on the distance between the surface and the molecule $d$. This is depicted in Fig. 2, where $d_{0}$ marks the equilibrium bond length between the nitrogen atoms of the bipyridine molecule [Fig. 2(a)] or the sulfur atoms of BPDT [Fig. 2(b)] and the Au atoms they are attached to. Since MO1 is $\sim 10 \mathrm{eV}$ below the lowest-lying Au valence states, its energetic position must be exclusively guided by rigid potential shifts without any direct hybridization effects. Now we want 

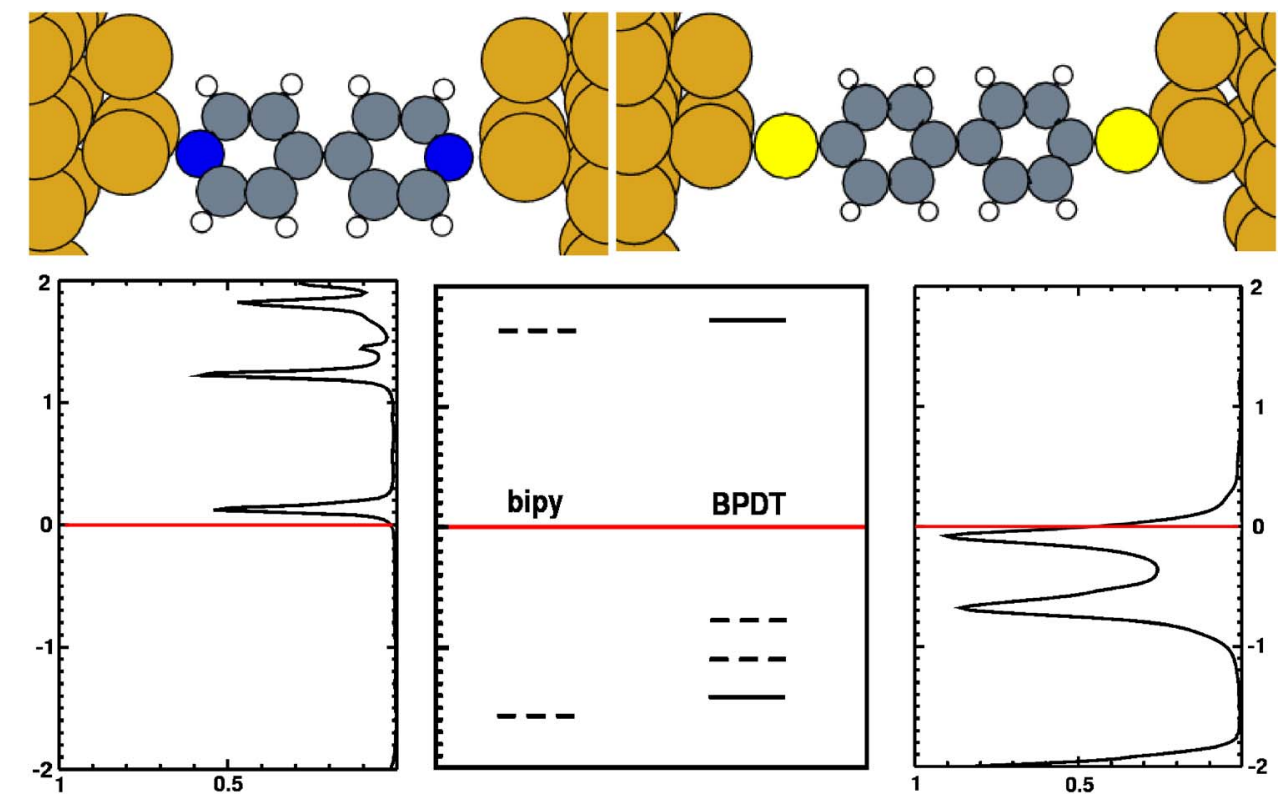

FIG. 1. (Color online) A bipyridine (left) and BPDT (right) molecule suspended between Au electrodes. The middle lower panel shows the alignment of the molecular levels with the metal Fermi energy [red (gray) line] by equalizing vacuum potentials of the isolated molecules and surfaces where the HOMO and LUMO are marked with dashed and the other MOs with solid lines. The left and right lower panels show transmission functions for the coupled systems for bipyridine and BPDT, respectively.

to address the question whether these rigid potential shifts have a quantifiable relation to charge being transferred between the molecule and the surface. In our study we make use of the concept of fractional charges. ${ }^{25}$ This makes it possible to determine the ground-state electron density and electronic eigenvalue spectrum for a (albeit only finite) system with fractions of electrons removed or added when compared to the total charge of all the nuclei. The shift of MO1 in the charged isolated molecules is shown as insets in Fig. 2 for bipyridine and BPDT. As can be seen the molecular levels move up when the molecule is charged as a consequence of electrostatic repulsion. Our main argument is that these level shifts of the free molecule can explain the level shifts in the coupled molecule-surface system. To show this we use the charge density differences between the coupled and isolated subsystems and the linear energy-charge relation for the free molecule. This allows us to calculate the position of MO1 as a function of distance, which we then compare directly with the actual energies from the coupled system in Fig. 2. For a physical interpretation of such fractional charges two notes of caution have to be made. (i) Fractional charges are only physically meaningful for small distances $d$, where an equilibrium between the molecule and the surface can be assumed. Our plots for long $d$ are only used for simulating a gradual switching on of the interaction. (ii) In our calculations we do not correct for the lack of the derivative discontinuity (DD) of the exchange correlation potential within standard DFT methods, which can lead to an underestimation of the gap between the highest occupied molecular orbital (HOMO) and LUMO and an overestimation in the conductance for weakly coupled single-molecule junctions even at low biases. ${ }^{26}$ Including DD would make our scheme impractical, since MO1 would jump discontinously when going from negative to positive charges. However, our study does not focus on absolute values for conductance or gap size but on the comparison of equilibrium charge transfer in two different junctions.

For bipyridine at very large distances $d$, MO1 should rest at an energetic position $\epsilon_{\text {vac-align }}$, which corresponds to the one it would hold if the vacuum levels of the isolated molecule and surface were aligned. Figure 2(a) indicates that for $d$ larger than $\sim 6 \AA$ there is little interaction between the two subsystems and that both are charge neutral within numerical precision. At the bonding distance $d_{0}$ an effective charge of -0.25 electrons on the bipyridine can be derived from the shift of MO1 by comparison with the charged free molecule. Partial charges with respect to the isolated subsystems can also be computed directly from electron density differences, which results in a charge of -0.23 electrons on the bipyridine molecule at the same distance. As can be seen from Fig. 1, the HOMO-LUMO gap is much smaller for BPDT $(\sim 0.3 \mathrm{eV})$ than for bipyridine $(\sim 3.2 \mathrm{eV})$. This is because BPDT lacks two electrons, which are subtracted from its $\pi$ system, when two hydrogen atoms are removed from the stable aromatic molecule biphenyl dithiol in order to form the highly reactive biradical BPDT, which is then attached to the Au surfaces. Since the molecular levels corresponding to the dangling bonds on the sulfur atoms are fully occupied in BPDT, the HOMO of biphenyl dithiol gets emptied and becomes the LUMO of BPDT. A further difference between bipyridine and BPDT (see Fig. 2) is that the long-distance position of MO1 for BPDT is not just $\epsilon_{\text {vac-align }}$ but is instead $\sim 0.5 \mathrm{eV}$ higher in energy. Vacuum level alignment for BPDT on Au (111) leads to a situation where the LUMO lies well below $E_{F}$ (see Fig. 1). Therefore, we find 0.11 electrons gain on the BPDT molecule from the MO1 level shifts and 0.09 from charge density differences $\Delta n\left(x_{z}\right)$ at large $d$. We stress again that such a transfer of fractional charges at long 

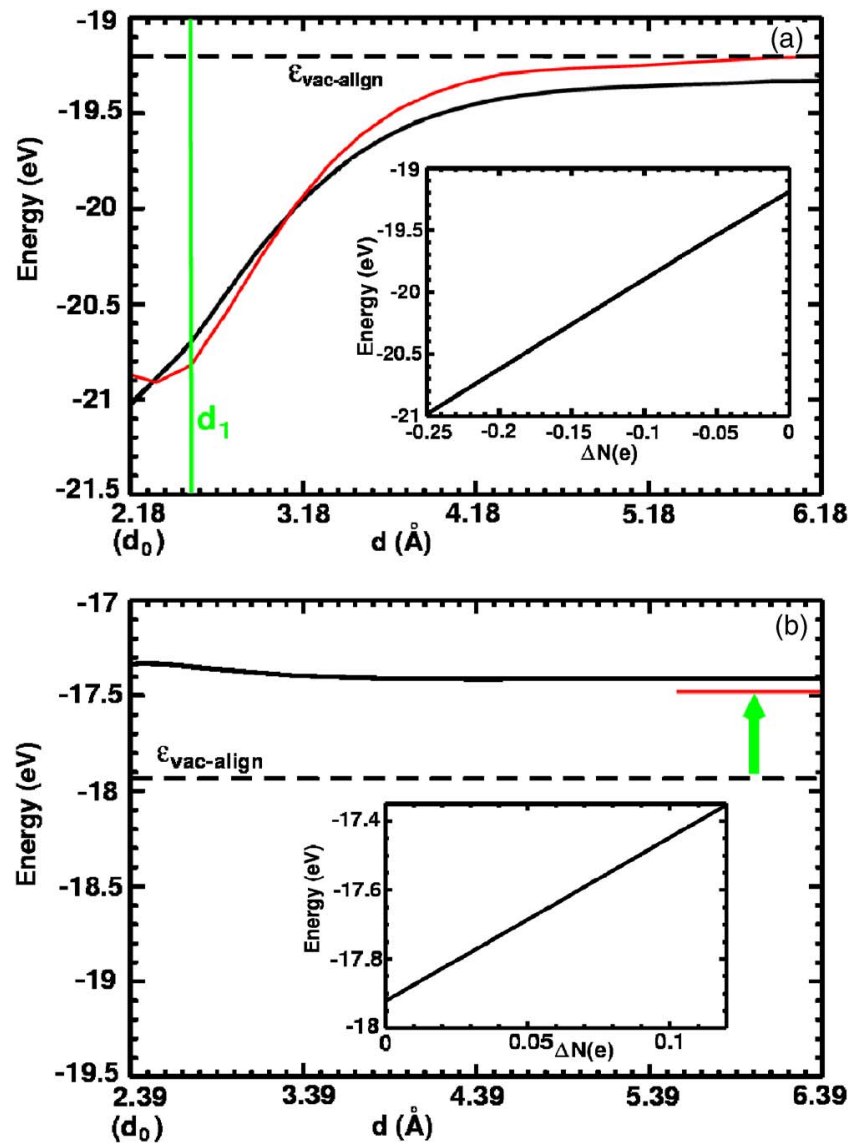

FIG. 2. (Color online) MO1 energies (relative to $E_{F}$ of the $\mathrm{Au}$ surface) depending on the distance $d$ between the molecule and the Au surfaces for (a) bipyridine and (b) BPDT. The black solid curves are taken from the coupled systems; for the gray (red) curves estimates from the charged free molecules have been used. The dashed line shows the position from vacuum alignment without charging $\left(\epsilon_{\text {vac-align }}\right)$, which was obtained from a free-molecule calculation and the work function of the metal slab. MO1 in its dependence on the charge for the free molecules is shown as insets.

distances is due to the lack of a DD in our standard DFT scheme. ${ }^{26}$ At the bonding distance $d_{0}$, the BPDT molecule gains 0.12 electrons calculated from MO1 level shifts and $\Delta n\left(x_{z}\right)$ cannot be interpreted unambigously due to the strong hybridization of $\mathrm{Au}$ and $\mathrm{S}$ states. The upward shift of the MOs in BPDT has been noticed before by Piccini et al. ${ }^{18}$ but interpreted differently.

An apparent problem with the picture we propose here is the following. In the case of bipyridine charge is moving away from the molecule and the levels are therefore shifting down in energy. However, as the LUMO hits $E_{F}$ it must begin to fill and this is naturally associated with charge being transferred to the molecule. Both effects are in fact taking place. For the further analysis of the bipyridine junction, we use a model system where the leads are replaced by single Au atoms. Looking at $\Delta n\left(x_{z}\right)$ in Fig. 3 we find maxima at the $\mathrm{Au}$ atoms and minima close to but not at the nitrogen positions. Nevertheless it can be seen from the integral (dashed line) that electrons are moving from the molecule to the surface. When we form charge density differences just using the

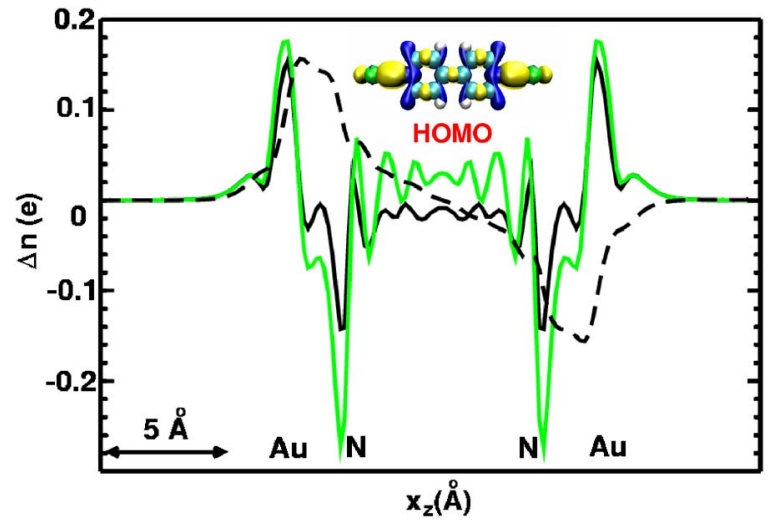

FIG. 3. (Color online) Charge density difference $\Delta n\left(x_{z}\right)$ (black solid line, summed up parallel to the surface plane) and its integral (black dashed line) for bipyridine coupled to $\mathrm{Au}$ atoms ( $d$ $=2.63 \AA$ ) $). \Delta n\left(x_{z}\right)$ constructed only from contributions of the bipyridine HOMO (see inset) and the Au $s$ and $d\left(z^{2}\right)$ states is also given for comparison [gray (green) line].

bipyridine HOMO (which is twice degenerate and both orbitals are fully occupied) and the Au $s$ and the also fully occupied $d\left(z^{2}\right)$ states, the result still has the same nodal structure as $\Delta n\left(x_{z}\right)$. The two functions differ in the minimum at the nitrogens being deeper for the difference constructed only from six orbitals. In the latter case there is also no charge depletion in the center of the molecule. Both aspects can be explained in terms of screening. Since a very localized minimum in the charge density is energetically unfavorable, the lower-lying MOs are polarized so that this minimum is at least partially smoothed out. Such a polarization of MOs has the effect that the total charge density at the center of the molecule is reduced, which explains the net charge transfer from the molecule to the Au. For the maximum at the $\mathrm{Au}$ atoms no such screening occurs since for the bare atoms there are no electrons available for achieving that. For the realistic surface calculations, however, the situation is different and the same peak in $\Delta n\left(x_{z}\right)$ (not shown here) becomes smaller and broader.

But what is the effect of the partial filling of the LUMO, which occurs in the bipyridine junction only for small $d$ ? It can be seen in the change of curvature in the MO1 energies vs. $d$ in Fig. 2(a) for distances smaller than $d_{1}$. Because now the process described in the last paragraph is partially neutralized by charge flowing back to the molecule, the lowering in energy of MO1 is slightly reduced, thereby flattening the curves. For Fig. 3 we have chosen a distance larger than $d_{1}$ in order to be able to discuss the two driving forces for charge movement separately. For BPDT the filling of the LUMO dominates, and the net effect is therefore a movement of electrons toward the molecule. The strong system dependence of the Fermi level alignment in molecular junctions can also be seen by a comparison of our results with recent articles on the adsorption of thiolates on $\mathrm{Au}(111),{ }^{27}$ where the main effect was found to be due to molecular dipoles which result from the asymmetry of the system.

In summary, we presented a detailed analysis of the energetic alignment of molecular orbitals with respect to the Fermi level of gold electrodes in single-molecule nanojunc- 
tions with bipyridine and biphenyl dithiolate molecules. The outcome of this alignment has a crucial effect on the zerobias conductance of the junction; the major source determining it is equilibrium charge transfer between the molecule and the electrodes. We established that a comparison between the energies of the lowest-lying molecular orbitals within the junction and for the isolated but partially charged molecule gives reasonable estimates for the net charge being transferred. For the bipyridine, the molecule is drained of electrons; the biphenyl dithiolate on the other hand gains electrons from the Au electrodes. From a simplified model of the junctions where the electrodes have been replaced by single $\mathrm{Au}$ atoms, we could derive that the charge transfer process for bipyridine is determined by the balance of two effects. Pauli repulsion between occupied molecular and $\mathrm{Au}$ orbitals pushes electrons away from the molecule and the interface region to more remote parts of the electrodes surface or bulk, which followed by screening depletes the molecule of electronic charge. The filling of the LUMO on the other hand results in an electron surplus on the molecule. For bipyridine the first effect dominates, for BPDT only the second effect can be unambigously observed. The presented scheme can also be used to analyze the effect of the local contact geometry on level alignment, which will be explored in a subsequent publication.

We appreciate useful discussions with Iben Kristensen, Mikkel Strange, and Kristian Thygesen. The Center for Atomic-Scale Materials Physics at NanoDTU is sponsored by the Danish National Research Foundation. We acknowledge support from the Nano-Science Center at the University of Copenhagen and from the Danish Center for Scientific Computing through Grant No. HDW-1101-05.
${ }^{1}$ H. Ohnishi, Y. Kondo, and K. Takayanagi, Nature (London) 344, 524 (1998)

${ }^{2}$ C. Joachim, J. K. Gimzewski, and A. Aviram, Nature (London) 408, 541 (2000).

${ }^{3}$ A. Aviram and M. A. Ratner, Chem. Phys. Lett. 29, 277 (1974).

${ }^{4}$ D. M. Eigler and E. K. Schweizer, Nature (London) 344, 56 (1991).

${ }^{5}$ C. Joachim, J. K. Gimzewski, R. R. Schlittler, and C. Chavy, Phys. Rev. Lett. 74, 2102 (1995).

${ }^{6}$ M. A. Reed, C. Zhou, C. J. Muller, T. P. Burgin, and J. M. Tour, Science 278, 252 (1997).

${ }^{7}$ J. Reichert, R. Ochs, D. Beckmann, H. B. Weber, M. Mayor, and H. v. Löhneysen, Phys. Rev. Lett. 88, 176804 (2002).

${ }^{8}$ R. H. M. Smit, Y. Noat, C. Untiedt, N. D. Lang, M. C. van Hemert, and J. M. van Ruitenbeek, Nature (London) 419, 906 (2002).

${ }^{9}$ Y. Fujimoto and K. Hirose, Phys. Rev. B 67, 195315 (2003).

${ }^{10}$ A. R. Rocha, V. M. García-Suárez, S. W. Baily, C. J. Lambert, J. Ferrer, and S. Sanvito, Nat. Mater. 4, 335 (2005).

${ }^{11}$ M. Brandbyge, J. L. Mozos, P. Ordejón, J. Taylor, and K. Stokbro, Phys. Rev. B 65, 165401 (2002).

${ }^{12}$ Y. Xue, S. Datta, and M. A. Ratner, Chem. Phys. 281, 151 (2002).

${ }^{13}$ A. Calzolari, N. Marzari, I. Souza, and M. B. Nardelli, Phys. Rev. B 69, 035108 (2004).

${ }^{14}$ L. V. Keldysh, Sov. Phys. JETP 20, 1018 (1965).

${ }^{15}$ Introducing Molecular Electronics, edited by G. Cuniberti, G. Fagas, and K. Richter (Springer, New York, 2005).

${ }^{16}$ R. Stadler, K. S. Thygesen, and K. W. Jacobsen, Phys. Rev. B 72, 241401(R) (2005).
${ }^{17}$ B. Xu and N. J. Tao, Science 301, 1221 (2003); B. Xu, X. Xiao, and N. J. Tao, J. Am. Chem. Soc. 125, 16164 (2003).

${ }^{18}$ Y. Xue, S. Datta, and M. A. Ratner, J. Chem. Phys. 115, 4292 (2001); Y. Xue and M. A. Ratner, Phys. Rev. B 68, 115406 (2003); S. Piccini, A. Selloni, S. Scandolo, R. Car, and G. Scoles, J. Chem. Phys. 119, 6729 (2003).

${ }^{19}$ B. Hammer, L. B. Hansen, and J. K. Nørskov, Phys. Rev. B 59, 7413 (1999); S. R. Bahn and K. W. Jacobsen, Comput. Sci. Eng. 4, 56 (2002); the Dacapo code can be downloaded at http:// www.fysik.dtu.dk/campos

${ }^{20}$ D. Vanderbilt, Phys. Rev. B 41, R7892 (1990).

${ }^{21}$ J. P. Perdew, J. A. Chevary, S. H. Vosko, K. A. Jackson, M. R. Pederson, D. J. Singh, and C. Fiolhais, Phys. Rev. B 46, 6671 (1992).

${ }^{22}$ Y. Meir and N. S. Wingreen, Phys. Rev. Lett. 68, 2512 (1992).

${ }^{23}$ K. S. Thygesen and K. W. Jacobsen, Chem. Phys. 319, 111 (2005); K. S. Thygesen, L. B. Hansen, and K. W. Jacobsen, Phys. Rev. Lett. 94, 026405 (2005); Phys. Rev. B 72, 125119 (2005).

${ }^{24}$ K. S. Thygesen and K. W. Jacobsen, Phys. Rev. B 72, 033401 (2005).

${ }^{25}$ M. E. Casida, Phys. Rev. B 59, 4694 (1999).

${ }^{26}$ C. Toher, A. Filippetti, S. Sanvito, and K. Burke, Phys. Rev. Lett. 95, 146402 (2005); M. Koentopp, K. Burke, and F. Evers, Phys. Rev. B 73, 121403(R) (2006).

${ }^{27}$ G. Heimel, L. Romaner, J. L. Bredas, and E. Zojer, Phys. Rev. Lett. 96, 196806 (2006); R. Rousseau, V. De Renzi, R. Mazzarello, D. Marchetto, R. Biagi, S. Scandolo, and U. del Pennino, J. Phys. Chem. B 110, 10862 (2006). 